\title{
Cryptococcus spp. em excretas de Columba livia (pombos domésticos) provenientes de um hospital universitário no Sul do Brasil
}

\author{
Cryptococcus spp. in excreta of Columba livia (domestic pigeons) \\ from a university hospital in southern Brazil
}

\author{
Cristiane Tavares de Lima', Gabriel Baracy Klafke', Melissa Orzechowski Xavier ${ }^{1 *}$

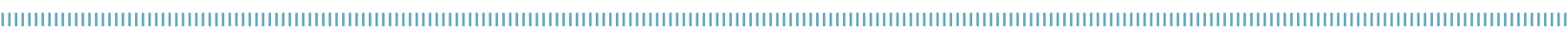

\begin{abstract}
RESUMO: A criptococose é uma micose primariamente pulmonar, sendo adquirida por meio de inalação de propágulos fúngicos infectantes encontrados no ambiente. Um dos principais agentes etiológicos da criptococose é a espécie Cryptococcus neoformans, que apresenta distribuição mundial. $\mathrm{O}$ objetivo desse trabalho foi pesquisar a ocorrência de Cryptococcus spp. em excretas de Columba livia (pombos domésticos) encontradas na área externa do prédio do Hospital Universitário Dr. Miguel Riet Correa Junior, centro de referência para o tratamento da Síndrome da Imunodeficiência Adquirida na regiáo sul do estado do Rio Grande do Sul, Brasil. Durante um período de dez meses, foi coletado um total de 40 amostras de excretas secas da área acadêmica e da área hospitalar do Hospital Universitário. As excretas foram adicionadas de solução salina com cloranfenicol, homogeneizados em vórtex, semeados em ágar Níger e incubados a $25^{\circ} \mathrm{C}$ com observaçóes diárias até sete dias. A identificação do micro-organismo foi realizada por provas fenotípicas e bioquímicas. Das 40 amostras processadas e analisadas, 13 (32,5\%) foram positivas para o isolamento de Cryptococcus spp., variando de 20.000 a 3.000.000 UFC/g de fezes. O isolamento desse fungo em um ambiente hospitalar é relevante em saúde pública, pois evidencia a exposiçáo dos indivíduos que frequentam esse local a propágulos infectantes.
\end{abstract}

PALAVRAS-CHAVE: Pombos, micose, criptococose, Cryptococcus spp., indivíduos imunossuprimidos.

\begin{abstract}
Cryptococcosis is primarily a pulmonary mycosis, being acquired by inhalation of infective fungal propagules found in the environment. One of the main etiological agents of cryptococcosis is the yeast Cryptococcus neoformans, which has worldwide distribution and whose natural habitat is the feces of birds. The aim of this study was to investigate the occurrence of Cryptococcus spp. in excreta of Columba livia (domestic pigeon) found in the outer area of the building of Hospital Universitário Dr. Miguel Riet Correa Junior, a reference center for the treatment of Acquired Immunodeficiency Syndrome in southern state of Rio Grande do Sul, Brazil. During a period of ten months, a total of 40 samples of dried excreta was collected from the site's academic area and hospital. To the excreta, were added saline solution with chloramphenicol, homogenized in a vortex, cultivated in Niger seed agar and incubated at $25^{\circ} \mathrm{C}$ with daily observations up to seven days. The identification of the microorganism was performed by phenotypical and biochemical tests. From the 40 samples processed and analyzed, $13(32.5 \%)$ were positive for the isolation of Cryptococcus spp., with the quantity of yeasts ranging from 20.000 to $3.000 .000 \mathrm{CFU} / \mathrm{g}$ of feces. Isolation of the opportunistic fungus Cryptococcus spp. at a university hospital is relevant in public health for demonstrating the exposure of individuals who frequent this site to infectant propagules.
\end{abstract}

KEYWORDS: Pigeons, mycosis, cryptococcosis, Cryptococcus spp., immunosuppressed individuals. 
Os fungos do gênero Cryptococcus sáo importantes micro-organismos oportunistas responsáveis pelo desencadeamento de uma micose primariamente pulmonar, e que apresentam tropismo para sistema nervoso central. Sáo fungos leveduriformes com colônias de cor branca a creme, brilhantes, com textura mucoide e margem lisa. $\mathrm{Na}$ micromorfologia, caracterizam-se por apresentar blastoconídios globosos ou ovalados e reproduçáo assexuada unibrotante, de colo estreito, os quais possuem uma cápsula de polissacarídeo (Kon et al., 2008).

Duas são as principais espécies patogênicas, $C$. neoformans e C. gattii, as quais se distinguem tanto por aspectos ecológicos, bioquímicos, antigênicos e genéticos (REOLON et al., 2004). O fungo C. neoformans tem distribuição mundial e seu habitat natural são as excretas de aves, especialmente de Columba livia (pombos domésticos) (Faria et al., 2010). Por outro lado, C. gattii ocorre em regióes tropicais e subtropicais, e tem como habitat natural os eucaliptos e material vegetal em decomposição. No Brasil, já foram isolados inclusive de árvores nativas, como as árvores da regiāo de Floresta Atlântica do estado de Espírito Santo (Ellis; Pfeiffer, 1990; Baltazar et al., 2008).

Quanto à patogenicidade, a espécie C. neoformans geralmente acomete os indivíduos com déficit imunitário, sendo considerada um agente oportunista (Darzé et al., 2000; Fernandes et al., 2000; Menezes et al., 2002; Moreira et al., 2006); enquanto que C. gattii é patógeno primário, podendo causar doença inclusive em indivíduos imunocompetentes (CorrêA et al., 1999; CorrêA et al., 2002). A criptococose é a micose causada por esses micro-organismos e ocorre a partir da inalação dos propágulos fúngicos que se dispersam pelo ar, sendo o pulmão o sítio anatômico primário da infecção (Reolon et al., 2004). Os quadros clínicos mais comuns desta micose são meningoencefalite (neurocriptococose), infecção pulmonar (criptococose pulmonar) e criptococose disseminada com fungemia (DARZÉ et al., 2000; Moreira et al., 2006).

Embora o C. neoformans atinja outros indivíduos imunodeprimidos como transplantados de órgãos, pacientes em tratamento com quimioterápicos ou em uso prolongado de corticosteroides, os indivíduos com a Síndrome da Imunodeficiência Humana Adquirida (SIDA) são os mais suscetíveis ao desencadeamento da doença, sendo considerados como o principal grupo de risco para criptococose (Fernandes et al., 2000; Menezes et al., 2002). Locais onde há acúmulo de excretas de aves servem como importante fonte de infecção do C. neoformans para estes indivíduos, aumentando seu risco de adquirir a doença devido a uma maior concentração de propágulos fúngicos infectantes presente nestes ambientes (Reolon et al., 2004). Sendo assim, este trabalho teve como objetivo pesquisar a ocorrência de
Cryptococcus spp. em excretas de C. livia encontradas no Hospital Universitário (HU) da Universidade Federal do Rio Grande (FURG - Campus Saúde).

O trabalho foi realizado no Campus Saúde da Universidade Federal do Rio Grande (HU-FURG), nas áreas acadêmica e hospitalar, de janeiro de 2011 a outubro de 2011. As amostras de excretas de C. livia foram coletadas semanalmente de locais como os parapeitos de janelas, as superfícies de ar condicionado e dos pátios internos na área acadêmica e hospitalar do Campus Saúde. As coletas foram realizadas das excretas secas, conforme descritos por diversos autores (Filiú et al., 2002; Reolon et al., 2004; BARoni et al., 2006; Contin et al., 2011), acondicionadas em frascos estéreis, transportadas ao Laboratório de Micologia da Faculdade de Medicina (FAMED-FURG) e processadas imediatamente.

Primeiramente, as excretas foram maceradas com auxilio de gral e pistilo e, na sequência, $1 \mathrm{~g}$ de amostra foi transferido para tubo estéril e homogeneizado por três minutos em $10 \mathrm{~mL}$ de solução salina estéril e $5 \mathrm{mg}$ de cloranfenicol, sendo posteriormente mantido em repouso por 30 minutos. O sobrenadante foi utilizado para realização de pour-plate com semeadura em ágar Níger (AN), utilizando alça de Drigalski estéril. O material foi incubado a $25^{\circ} \mathrm{C}$ por até sete dias com observaçáo diária para detecção de crescimento fúngico.

Após incubação, as colônias leveduriformes compatíveis com Cryptococcus spp., de consistência mucoide, brilhantes e que adquiriram uma coloração marrom, foram submetidas ao exame micromorfológico para identificação da presença da cápsula de mucopolissacarídio ao redor de blastoconídios redondos a ovalados, característica típica de fungos do gênero Cryptococcus. Este exame foi realizado dispondo uma alçada da colônia juntamente com a tinta da China (nigrosina), com posterior visualizaçáo em microscopia óptica. As colônias de Cryptococcus spp. foram repicadas em tubos com ágar ureia base (Christensen), e incubadas a $25^{\circ} \mathrm{C}$ por cinco dias para comprovação da produção de urease pela levedura, e em tubos com ágar CGB (Canavanina Glicina Azul de Bromotimol) para diferenciação da espécie C. gattii.

Após a identificação da levedura, foi realizada a contagem do total de unidades formadoras de colônias (UFC) típicas de Cryptococcus nas placas. Considerando que cada grama de excreta foi homogeneizada em $10 \mathrm{~mL}$, e que $0,1 \mathrm{~mL}$ foi o volume utilizado para semeadura das amostras, o valor obtido foi multiplicado por 100 e pela diluiçấo previamente realizada, para expressar um resultado final em UFC/grama de excreta. Os dados foram computados e analisados estatisticamente com o auxílio do programa SPSS 16.0, utilizando o teste qui-quadrado com nível de significância de 5\%. 
Durante o período do estudo, foram coletadas no HU-FURG um total de 40 amostras de excretas secas de C. livia. Destas, 21 foram coletadas da área acadêmica e 19 da área hospitalar, sendo 12 amostras de parapeitos de janela, 21 dos pátios internos e sete das superfícies de ar-condicionado.

Das 40 amostras analisadas, 13 (32,5\%) foram positivas para isolamento de fungos do gênero Cryptococcus. Das 27 restantes, $11(40,7 \%)$ amostras foram negativas e 16 $(59,3 \%)$ amostras foram consideradas contaminadas devido ao crescimento rápido e abundante de fungos ambientais, principalmente zigomicetos, impedindo a visualização das demais colônias fúngicas isoladas.

Todos os isolados leveduriformes identificados como pertencentes ao gênero Cryptococcus apresentaram colônias de consistência mucoide, brilhantes e de coloração branca a bege em ágar Sabouraud dextrose com cloranfenicol, e marrom no ágar Níger, sendo observada na micromorfologia a presença de blastoconídios redondos a ovalados com cápsula de polissacarídeo ao seu redor. Todos apresentaram urease positiva e CGB negativos.

A quantidade de UFC/g de excretas variou de 20.000 a 3.000.000 (média: 344.167; mediana: 20.000). Não foi encontrada diferença significativa no isolamento de Cryptococcus spp. das excretas quanto ao local procedente (área hospitalar ou área acadêmica; $\mathrm{p}=0,194$ ) e quanto à superfície de origem (parapeitos de janela, chão ou superfície de ar-condicionado; $\mathrm{p}=2,68)$.

O presente trabalho avaliou a presença de fungos do gênero Cryptococcus em excretas de pombos domésticos (C. livia) provenientes de um ambiente hospitalar e acadêmico. O isolamento de Cryptococcus spp. ocorreu em $32,5 \%$ das amostras, valores semelhantes aos encontrados por BARONi et al. (2006) e FAria et al. (2010), que isolaram o fungo em $37,8 \%$ e $27 \%$ das amostras de excretas de pombos coletadas de prédios urbanos, respectivamente. Por outro lado, em outro ambiente pesquisado, Reolon et al. (2004) isolaram o fungo em $100 \%$ das amostras coletadas em praças ao ar livre. Esta diferença pode ser justificada pelo fato de que as amostras do nosso estudo foram coletadas de superfícies e não diretamente dos ninhos dos pombos domésticos, locais de grandes acúmulos de excretas, o que favorece a proliferação fúngica (CAICEDo et al., 1996).

Excluindo as amostras contaminadas $(\mathrm{n}=16)$, que não puderam ser analisadas quanto à presença da levedura pesquisada, obteve-se um total de 24 amostras, sendo 13 (54\%) positivas para o isolamento de Cryptococcus spp. e 11 (46\%) negativas. Neste caso, obteve-se mais da metade de amostras positivas para o isolamento de fungos do gênero Cryptococcus, tornando estes dados ainda mais preocupantes por se tratar de um ambiente de grande circulação de pessoas. Não foram encontrados outros trabalhos similares realizados em prédios de hospitais na literatura, sendo este o único estudo realizado em local de ambiente hospitalar, mostrando a presença de fontes ambientais de fungos do gênero Cryptococcus, como consequência do acúmulo de excretas de C. livia, o que demonstra a exposição de inúmeras pessoas, desde estudantes, docentes, técnicos, pacientes (especialmente imunossuprimidos altamente susceptíveis à infecção) e acompanhantes aos propágulos infectantes do fungo.

Conforme os dados da literatura o habitat natural do C. neoformans se caracteriza por excretas de aves, especialmente de pombos domésticos ( $C$. livia), embora já tenha sido isolado de fezes de outras aves, como Melopsittacus undulatus (periquito australiano), Columba sp. (pombo rabo de leque), Serinus canaria (canário belga), Taeniopigya guttata (mandarim), Agapornis sp. (agapore) e Nymphicus hollandicus (calopsita) (Filıú et al., 2002). A facilidade do desenvolvimento da levedura nesses ambientes se deve ao fato de que este fungo utiliza a creatina, o ácido úrico, as purinas e xantinas presentes nas excretas como fonte de nitrogênio para sua sobrevivência e reprodução (Levitz et al., 1991).

Em uma amostra de excreta positiva do HU-FURG, não foi possível a contagem das UFC; em sete amostras, foi observado o crescimento de 20.000 UFC de Cryptococcus spp./grama de excreta, e nas outras cinco amostras positivas, foi observado o crescimento de 3.000.000, 600.000, 250.000, 90.000 e 50.000 UFC/grama. Estes valores foram mais altos do que os já descritos na literatura por Reolon et al. (2004), que isolaram mais de 4.000 UFC em uma amostra e mais de 10.000 unidades formadoras de colônias/grama de material semeado nas outras amostras. Porém, as sete amostras com 20.000 UFC se aproximaram dos valores descritos por Contin et al. (2011), que isolaram até 20.000 UFC/grama de material semeado em suas amostras. Já FiLıú et al. (2002), estudando excretas de aves de cativeiro, isolaram de 900 UFC de C. neoformans a 46.200 UFC por grama de material semeado em dez amostras, sendo oito com até 12.000 UFC, uma amostra com 25.000 UFC e uma amostra com 46.200 UFC, valores aproximados dos encontrados nesse trabalho.

A levedura do gênero Cryptococcus está presente em excretas de C. livia provenientes da área hospitalar e acadêmica pertencentes ao Campus Saúde (HU-FURG). A presença desse agente é preocupante devido à exposição de discentes, docentes, profissionais da saúde e principalmente pacientes imunocomprometidos, que correspondem a um grupo de risco para desenvolver a criptococose. Dessa forma, é necessária a precaução a partir de medidas de controle das aves, especialmente controle de sua população, a fim de prevenir a transmissão de doenças e a disseminação no ambiente do agente. 
BALTAZAR, L.M.; RIBEIRO, M.A. Primeiro isolamento ambiental de Cryptococcus gattii no Estado do Espírito Santo. Revista da Sociedade Brasileira de Medicina Tropical, v.41, n.5, p.449453, 2008.

BARONI, F.A.; PAULA, C.R.; SILVA É.G.; VIANI, F.C.; RIVERA, I.N.G.; OLIVEIRA, M.T.B.; GAMBALE, W. Cryptococcus neoformans strains isolated from church towers in Rio de Janeiro city, RJ, Brazil. Revista do Instituto de Medicina Tropical de São Paulo, v.48, n.2, p.71-75, 2006.

CAICEDO, L.D.; ALVAREZ, M.I.; LLANOS, C.E.E.; MOLINA, D. Cryptococcus neoformans en excretas de palomas del perímetro urbano de Cali. Colombia Médica, v.27, n.3-4, p.106-109, 1996.

CASADEVALL, A.; STEENBERGEN, J.N.E.; NOSANCHUK, J.D. 'Ready made' virulence and 'dual use' virulence factors in pathogenic environmental fungi - the Cryptococcus neoformans paradigm. Current Opinion in Microbiology, v.6, n.4, p.332337, 2003.

CONTIN, J.T.; QUARESMA, G.S.; SILVA, E.F.E.; LINARDI, V.R. Ocorrência de Cryptococcus neoformans em fezes de pombos na cidade de Caratinga, MG - Brasil. Revista Médica de Minas Gerais, v.21, n. 1, p. 19-24, 2011.

CORRÊA, M.P.S.C.; OLIVEIRA, E.C.; DUARTE, R.R.B.S.; PARDAL, P.P.O.; OLIVEIRA, F.M.; SEVERO, L.C. Criptococose em crianças no Estado do Pará, Brasil. Revista da Sociedade Brasileira de Medicina Tropical, v.32, n.5, p.505-508, 1999.

CORREAA, M.P.S.C.; SEVERO, L.C.; OLIVEIRA, F.M.; IRION, K.; LONDERO, A.T. The spectrum of computerized tomography (CT) findings in central nervous system (CNS) infection due to Cryptococcus neoformans var. gattii in immunocompetent children. Revista do Instituto de Medicina Tropical de São Paulo, v.44, n.5, p.283-287, 2002.

DARZÉ, C.; LUCENA, R.; GOMES, I.; MELO, A. Características clínicas laboratoriais de 104 casos de meningoencefalite criptocócica. Revista da Sociedade Brasileira de Medicina Tropical, v.33, n.1, p.21-26, 2000.

DUARTE, A.; ORDOÑEZ, N.; CASTAÑEDA, E. Asociacion de leveduras del genero Cryptococcus con especies de Eucalyptus en Santafe de Bogota. Revista do Instituto de Medicina Tropical de São Paulo, v.36, n.2, p.125-130, 1994.
ELLIS, D.H.; PFEIFFER, T.J. Natural Habitat of Cryptococcus neoformans var. gattii. Journal of Clinical Microbiology, v.28, n.7, p.1642-1644, 1990.

FARIA, R.O.; NASCENTE, P.S.; MEINERZ, A.R.M.; CLEFF, M.B.; ANTUNES, T.A.; SILVEIRA, É.S.; NOBRE, M.O.; MEIRELES, M.C.A.; MELLO, J.R.B. Ocorrência de Cryptococcus neoformans em excretas de pombos na Cidade de Pelotas, Estado do Rio Grande do Sul. Revista da Sociedade Brasileira de Medicina Tropical, v.43, n.2, p. 198-200, 2010.

FERNANDES, O.F.L.; COSTA, T.R.; COSTA, M.R.; SOARES, A.J.; PEREIRA, A.J.S.C.; SILVA, M.R.R. Cryptococcus neoformans isolados de pacientes com AIDS. Revista da Sociedade Brasileira de Medicina Tropical, v.33, n. 1, p.75-78, 2000.

FILIÚ, W.F.O.; WANKE, B.; AGÜENA, S.M.; VILELA, V.O.; MACEDO, R.C.L.; LAZÉRA, M. Cativeiro de aves como fonte de Cryptococcus neoformans na cidade de Campo Grande, Mato Grosso do Sul, Brasil. Revista da Sociedade Brasileira de Medicina Tropical, v.35, n.6, p.591-595, 2002.

KON, A.S.; GRUMACH, A.S.; COLOMBO, A.L.; PENALVA, A.C.O.; WANKE, B.; TELLES, F.Q.; SEVERO, L.C.; ARANHA, L.F.; LAZÉRA, M.S.; RESENDE, M.R.; SALMITO, M.A.; SHIKANAI-YASUDA, M.A.; MORETTI, M.L.; FERREIRA, M.S.; SILVA-VERGARA, M.L.; ANDRADE, N.M.P.; TRABASSO, P.; MENDES, R.P.; MARTINEZ, R.; PONZIO, V. Consenso em Criptococose - 2008. Revista da Sociedade Brasileira de Medicina Tropical, v.41, n.5, p.524-544, 2008.

LEVITZ, S.M. The ecology of Cryptococcus neoformans and the epidemiology of cryptococcosis. Reviews of Infectious Diseases, v.13, n.6, p.1163-1169, 1991.

MENEZES, E.A.; MONTEIRO, M.N.R.; ANGELO, M.R.F.; SANTOS, C.D.; FREIRE, C.C.F.; CUNHA, F.A. Cryptococcus neoformans causing meningitis in AIDS patients. Revista da Sociedade Brasileira de Medicina Tropical, v.35, n.5, p.537-539, 2002.

MOREIRA, T.A.; FERREIRA, M.S.; RIBAS, R.M.; BORGES, A.S. Criptococose: estudo clínico-epidemiológico, laboratorial e das variedades do fungo em 96 pacientes. Revista da Sociedade Brasileira de Medicina Tropical, v.39, n.3, p.255-258, 2006.

REOLON, A.; PEREZ, L.R.R.; MEZZARI, A. Prevalência de Cryptococcus neoformans nos pombos urbanos da Cidade de Porto Alegre, Rio Grande do Sul. Jornal Brasileiro de Patologia e Medicina Laboratorial, v.40, n.5, p.293-298, 2004. 